\title{
Melting Pattern of Diquark Condensates in Quark Matter
}

\author{
K. Iida ${ }^{1}$ T. Matsuura, ${ }^{2}$ M. Tachibana,${ }^{1}$ and T. Hatsuda ${ }^{2}$ \\ 1 The Institute of Physical and Chemical Research (RIKEN), Wako, Saitama 351-0198, Japan \\ ${ }^{2}$ Department of Physics, University of Tokyo, Tokyo 113-0033, Japan
}

\begin{abstract}
Thermal color superconducting phase transitions in high density three-flavor quark matter are investigated in the Ginzburg-Landau approach. Effects of nonzero strange quark mass, electric and color charge neutrality, and direct instantons are considered. Weak coupling calculations show that an interplay between the mass and electric neutrality effects near the critical temperature gives rise to three successive second-order phase transitions as the temperature increases: a modified colorflavor locked (mCFL) phase ( $u d, d s$, and $u s$ pairings) $\rightarrow$ a "dSC" phase ( $u d$ and $d s$ pairings) $\rightarrow$ an isoscalar pairing phase ( $u d$ pairing) $\rightarrow$ a normal phase (no pairing). The dSC phase is novel in the sense that while all eight gluons are Meissner screened as in the mCFL phase, three out of nine quark quasiparticles are gapless.
\end{abstract}

PACS numbers: 12.38.-t,12.38.Mh,26.60.+c

Unraveling the phase structure at high baryon density is one of the most challenging problems in quantum chromodynamics (QCD). Among others, color superconductivity in cold dense quark matter has been discussed from various viewpoints 1 , 2]. In relation to real systems such as newly born compact stars in stellar collapse, it is important to study the color superconductivity not only as a function of the quark chemical potential $\mu$ but also as a function of the temperature $T$. This is because the possible presence of color superconducting quark matter in a star affects the star's thermal evolution [3].

The purpose of this Letter is to investigate phase transitions in color superconducting quark matter with three flavors $(u d s)$ and three colors $(R G B)$ near the transition temperatures. We consider a realistic situation in which nonzero strange quark mass $m_{s}$, electric and color charge neutrality, and direct instantons take effect. As we shall see in weak coupling $\left(m_{s}, \Lambda_{\mathrm{QCD}} \ll \mu\right)$, the effects of nonzero $m_{s}$ and electric neutrality are important in that they induce multiple phase transitions that change the pattern of diquark pairing as $T$ increases. In particular, we find a new phase, which we call "dSC," as an interface between a modified type of color-flavor locked (mCFL) phase and an isoscalar two-flavor (2SC) phase.

Throughout this Letter, we adopt the GinzburgLandau (GL) approach near the transition temperatures, which was previously used to study the massless threeflavor case [4, 5, [6] and is a more advantageous framework to weak coupling calculations than other mean-field approaches 7, 8]. In a realistic situation, the GL potential acquires the following corrections. First of all, nonzero $m_{s}$ affects the potential through the $s$ quark propagator 8] in such a way as to lower the temperature at which a diquark condensate with $s$ quarks dissolves. This is because the pairing interaction due to one-gluon exchange is effectively diminished by $m_{s}$ if the pair contains the $s$ quark. Secondly, when quark matter with nonzero $m_{s}$ is beta equilibrated and neutralized by electrons near the transition temperatures, the chemical potentials between $d, s$ quarks and $u$ quarks differ. Through this chemical potential difference, the GL potential acquires another $m_{s}$ dependence, which is the essential origin of the dSC phase. Thirdly, the instanton contribution gives an $m_{s}$ dependence to the GL potential through the effective four-fermion interaction proportional to $m_{s}$. We finally note that in weak coupling, color neutrality makes negligible difference in the GL potential near the transition temperatures [4].

We assume that diquark pairing takes place in the color-flavor antisymmetric channel with $J^{P}=0^{+}$, which is predicted to be the most attractive channel in weak coupling [9]. In this case, the pairing gap of a quark of color $b$ and flavor $j$ with that of color $c$ and flavor $k$ has the form $\phi_{b c j k}=\epsilon_{a b c} \epsilon_{i j k}\left(\mathbf{d}_{a}\right)^{i}$ [4]. Here the $3 \times 3$ matrix $\left(\mathbf{d}_{a}\right)^{i}$ transforms as a vector under $G=S U(3)_{C} \times S U(3)_{L+R} \times U(1)_{B}$ and belongs to the $\left(3^{*}, 3^{*}\right)$ representation of $S U(3)_{C} \times S U(3)_{L+R}$.

For a homogeneous system of massless quarks $\left(m_{u, d, s}=0\right)$, the GL potential is invariant under $S U(3)_{C} \times S U(3)_{L+R}$ and valid near the critical temperature, $T_{c}$, common to all states belonging to the channel considered here. This potential reads [4, 10]

$$
S=\bar{\alpha} \sum_{a}\left|\mathbf{d}_{a}\right|^{2}+\beta_{1}\left(\sum_{a}\left|\mathbf{d}_{a}\right|^{2}\right)^{2}+\beta_{2} \sum_{a b}\left|\mathbf{d}_{a}^{*} \cdot \mathbf{d}_{b}\right|^{2}
$$

where $\left(\mathbf{d}_{a}\right)^{i}=\left(d_{a}^{u}, d_{a}^{d}, d_{a}^{s}\right)$ and the inner product is taken for flavor indices. In the weak coupling regime, the coefficients are [4]

$$
\beta_{1}=\beta_{2}=\frac{7 \zeta(3)}{8\left(\pi T_{c}\right)^{2}} N(\mu) \equiv \beta, \quad \bar{\alpha}=4 N(\mu) t \equiv \alpha_{0} t,
$$

where $N(\mu)=\mu^{2} / 2 \pi^{2}$ is the density of states at the Fermi surface, and $t=\left(T-T_{c}\right) / T_{c}$ is the reduced temperature. With the parameters (2), one finds a second order phase transition at $T=T_{c}$ from the CFL phase $\left(d_{a}^{i} \propto \delta_{a}^{i}\right)$ to the normal phase $\left(d_{a}^{i}=0\right)$ in mean-field theory [4].

Let us now consider the effect of a nonzero $m_{s}$ in the quark propagator on the GL potential. We assume 
$m_{u, d}=0$ for simplicity and consider the high density regime, $m_{s} \ll \mu$. Near $T_{c}$ the leading effect of $m_{s}$ is to modify the quadratic term in the GL potential (11). The corrections to the quartic terms are subleading and negligible. Since $m_{s}$ affects only $u s$ and $d s$ pairings, the correction to the quadratic term has the form

$$
\epsilon \sum_{a}\left(\left|d_{a}^{u}\right|^{2}+\left|d_{a}^{d}\right|^{2}\right)=\epsilon \sum_{a}\left(\left|\mathbf{d}_{a}\right|^{2}-\left|d_{a}^{s}\right|^{2}\right) .
$$

Note that this correction induces an asymmetry in the flavor structure of the CFL phase.

In weak coupling, $\epsilon$ can be calculated by including $m_{s}$ in the Nambu-Gor'kov quark propagator when evaluating the thermodynamic potential. Following Ref. [4], we expand the thermodynamic potential not only in $d_{a}^{i}$ but also in $m_{s}$ up to $\mathcal{O}\left(m_{s}^{2}\right)$, and obtain

$$
\epsilon \simeq \alpha_{0} \frac{m_{s}^{2}}{4 \mu^{2}} \ln \left(\frac{\mu}{T_{c}}\right) \sim 2 \alpha_{0} \sigma .
$$

Here the dimensionless parameter $\sigma$ is given by

$$
\sigma=\left(\frac{3 \pi^{2}}{8 \sqrt{2}}\right) \frac{m_{s}^{2}}{g \mu^{2}}
$$

where $g$ is the QCD coupling constant. As long as $\sigma \ll 1$, which is relevant at asymptotically high density, the following GL analysis near $T_{c}$ is valid. In the latter estimate in Eq. (4) we use the leading-order result in $g$, $\ln \left(T_{c} / \mu\right) \sim-3 \pi^{2} /(\sqrt{2} g)$ [9]. This behavior of $T_{c}$ originates from the long-range color magnetic interaction which prevails in the relativistic regime. As a result of the modification by $m_{s}$ to $\ln \left(T_{c} / \mu\right), \epsilon$ has a positive value such that $u d$ pairing is favored over us and $d s$ pairings. Consequently, the CFL phase becomes asymmetric in flavor space and its critical temperature is lowered, leading to the appearance of the $2 \mathrm{SC}$ phase $\left(d_{a}^{i} \propto \delta^{i s}\right)$ just below $T_{c}$ [8]. Note that Eq. (3) has no effect on the 2SC phase. We also note that $T_{c}$ itself is modified by $m_{s}$ through the modification of the normal medium as $T_{c}(1+\mathcal{O}(g \sigma))$.

We turn to discuss effects of charge neutrality which also depend on $m_{s}$ as mentioned above. Under beta equilibrium and charge neutrality, the electron chemical potential $\mu_{e}$ and the shift $\delta \mu_{i}$ of the chemical potential of flavor $i$ from the average $(\mu)$ are related by $\delta \mu_{i}=-q_{i} \mu_{e}$, with electric charge $q_{i}$. We see from Ref. [4] that the thermodynamic-potential correction due to $\delta \mu_{i}$ has the form

$$
\eta\left(\frac{1}{3} \sum_{a}\left|\mathbf{d}_{a}\right|^{2}-\sum_{a}\left|d_{a}^{u}\right|^{2}\right)
$$

In weak coupling, where one may regard normal quark matter and electrons as noninteracting Fermi gases, $\mu_{e}$ is related to $m_{s}$ as $\mu_{e}=m_{s}^{2} / 4 \mu$. This estimate is valid in the vicinity of $T_{c}$ where corrections to $\mu_{e}$ by a finite pairing gap affect only the quartic terms in the GL potential. By combining this estimate with the weak coupling expression for $\eta / \mu_{e}$ given in Ref. [4], we obtain

$$
\eta \simeq \alpha_{0} \frac{m_{s}^{2}}{8 \mu^{2}} \ln \left(\frac{\mu}{T_{c}}\right) \sim \alpha_{0} \sigma .
$$

Since $\eta>0, d s$ pairing is more favorable than $u d$ and $u s$ pairings. This feature stems from the modification by $\delta \mu_{i}$ to the exponential factor of $T_{c}, \exp \left[-3 \pi^{2} /(\sqrt{2} g)\right]$, which tends to increase (decrease) the critical temperature for ij pairing when $\delta \mu_{i}+\delta \mu_{j}>0(<0)$.

We consider color neutrality of the system as well. In contrast to the case at $T=0$, however, it affects only the quartic terms in the GL potential through possible chemical potential differences between colors [4, 11], and in weak coupling its magnitude is suppressed by $\mathcal{O}\left(\left(T_{c} / g \mu\right)^{2}\right)$ compared to the leading quartic terms. Thus color neutrality has no essential consequence to the phase transitions considered in this Letter. A major difference between corrections of the charge neutrality and the color neutrality is that the former shifts the quark chemical potentials even in the normal phase, while the latter works only when the pairing occurs. This is why the former is more important than the latter near $T_{c}$.

The direct instanton at nonzero $m_{s}$, which induces an effective four-fermion interaction between $u$ and $d$ quarks 12], leads to a quadratic term in the GL potential, $\zeta \sum_{a}\left|d_{a}^{s}\right|^{2}$. An explicit weak coupling calculation shows that $\zeta \sim-\alpha_{0}\left(m_{s} / \mu\right)\left(\Lambda_{\mathrm{QCD}} / \mu\right)^{9}(1 / g)^{14}$. The negative sign indicates that the instanton favors $u d$ pairing as does one-gluon exchange [see Eq. (3)], but the magnitude of $\zeta$ is highly suppressed at high densities. Hereafter we will thus ignore instanton effects.

Since the two effects of nonzero $m_{s}$, characterized by Eqs. (3) and (6), favor $u d$ pairing and $d s$ pairing, respectively, the finite temperature transition from the CFL to the normal phase at $m_{s}=0$ is expected to be significantly modified. In fact, successive color-flavor unlocking transitions take place instead of a simultaneous unlocking of all color-flavor combinations. To describe this hierarchical thermal unlocking, it is convenient to introduce a parameterization,

$$
d_{a}^{i}=\left(\begin{array}{lll}
\Delta_{1} & 0 & 0 \\
0 & \Delta_{2} & 0 \\
0 & 0 & \Delta_{3}
\end{array}\right),
$$

where $\Delta_{1,2,3}$ are assumed without loss of generality to be real. We also name the phases for later convenience as

$$
\begin{aligned}
& \Delta_{1,2,3} \neq 0 \quad: \text { mCFL, } \\
& \Delta_{1}=0, \quad \Delta_{2,3} \neq 0: \text { uSC, } \\
& \Delta_{2}=0, \quad \Delta_{1,3} \neq 0: \mathrm{dSC}, \\
& \Delta_{1,2}=0, \quad \Delta_{3} \neq 0 \quad: 2 \mathrm{SC},
\end{aligned}
$$

where dSC (uSC) stands for superconductivity in which for $d(u)$ quarks all three colors are involved in the pairing. 


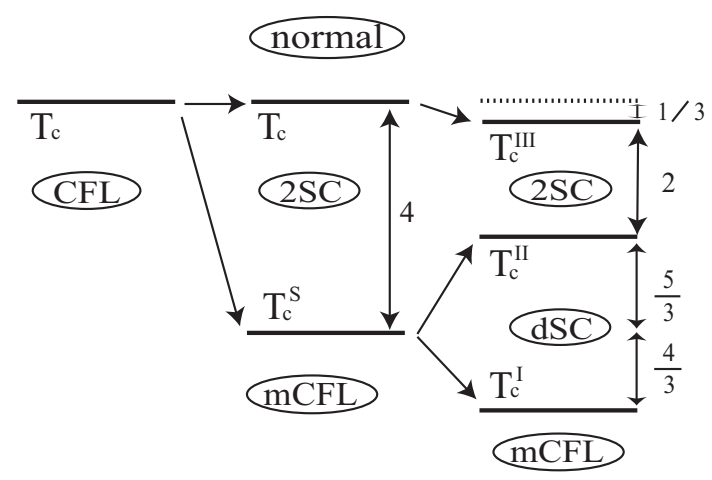

(a)

(b)

(c)

FIG. 1: Transition temperatures of the three-flavor color superconductor in weak coupling: (a) all quarks are massless; (b) nonzero $m_{s}$ in the quark propagator is considered; (c) electric charge neutrality is further imposed. The numbers attached to the arrows are in units of $\sigma T_{c}$.

In terms of the parameterization (8), the GL potential with corrections of $\mathcal{O}\left(m_{s}^{2}\right)$ to the quadratic term, Eqs. (31) and (6), reads

$$
\begin{aligned}
S & =\bar{\alpha}^{\prime}\left(\Delta_{1}^{2}+\Delta_{2}^{2}+\Delta_{3}^{2}\right)-\epsilon \Delta_{3}^{2}-\eta \Delta_{1}^{2} \\
& +\beta_{1}\left(\Delta_{1}^{2}+\Delta_{2}^{2}+\Delta_{3}^{2}\right)^{2}+\beta_{2}\left(\Delta_{1}^{4}+\Delta_{2}^{4}+\Delta_{3}^{4}\right)
\end{aligned}
$$

where $\bar{\alpha}^{\prime}=\bar{\alpha}+\epsilon+\frac{\eta}{3}$.

We proceed to analyse the phase structure dictated by Eq. (10) with the weak coupling parameters (2), (4), and (7) up to leading order in $g$. In Figs. 1 and 2 the results obtained by solving the coupled algebraic equations, $\partial S / \partial \Delta_{1,2,3}=0$, are summarized. Figure $1(\mathrm{a})$ shows the second-order phase transition, CFL $\rightarrow$ normal for $m_{s}=0$. Figures $1(\mathrm{~b}, \mathrm{c})$ represent how the phase transitions and their critical temperatures bifurcate as we introduce (b) effects of a nonzero $m_{s}$ in the quark propagator and then (c) effects of charge neutrality. In case (b), two second-order phase transitions arise, mCFL (with $\left.\Delta_{1}=\Delta_{2}\right) \rightarrow 2 \mathrm{SC}$ at $T=T_{c}^{s} \equiv(1-4 \sigma) T_{c}$, and $2 \mathrm{SC} \rightarrow$ normal at $T=T_{c}$. In case (c), there arises three successive second-order phase transitions, $\mathrm{mCFL} \rightarrow \mathrm{dSC}$ at $T=T_{c}^{\mathrm{I}}, \mathrm{dSC} \rightarrow 2 \mathrm{SC}$ at $T=T_{c}^{\mathrm{II}}$, and $2 \mathrm{SC} \rightarrow$ normal at $T=T_{c}^{\mathrm{III}}$. Shown in Fig. 2 is the $T$-dependence of the gaps $\Delta_{1,2,3}$ for case (c). All the gaps are continuous functions of $T$, but their slopes are discontinuous at the critical points, which reflects the second order nature of the transitions in the mean-field treatment of Eq. (10).

We may understand the bifurcation of the transition temperatures in weak coupling as follows. In the massless case (a), $T_{c}$ is degenerate between the CFL and $2 \mathrm{SC}$ phases, the chemical potential is common to all three flavors and colors, and the CFL phase is more favorable than the 2SC phase below $T_{c}$. As one goes from (a) to (b), nonzero $m_{s}$ sets in, which tends to suppress the pairing interactions including the $s$ quark. The critical temperature for the CFL phase is then lowered, which

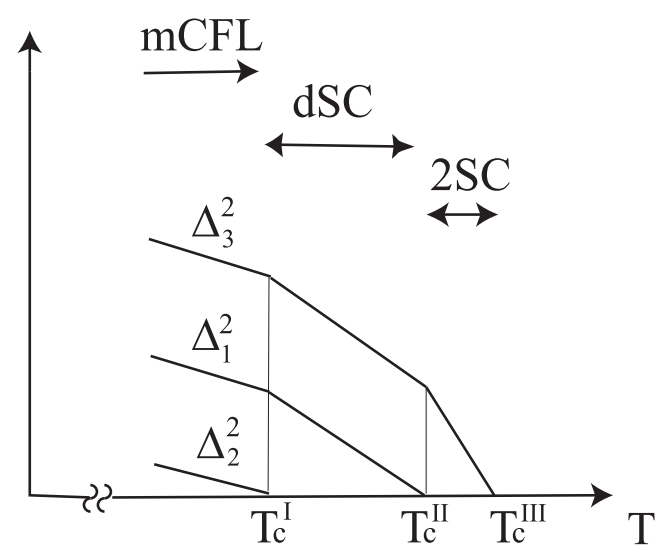

FIG. 2: A schematic illustration of the gaps squared as a function of $T$.

allows the 2SC phase to appear at temperatures between $T_{c}^{s}$ and $T_{c}$. As one goes from (b) to (c), charge neutrality sets in, which acts to decrease the chemical potential of $u$ quarks by $(2 / 3) \mu_{e}$ and increase that of $d$ and $s$ quarks by $(1 / 3) \mu_{e}$. Since $\mu_{e}>0$, the average chemical potential of quarks involved in $d s$ pairing increases, while those in $u d$ and us pairings decrease equally. Accordingly, the transition temperatures further change from $T_{c}$ to $T_{c}^{\mathrm{III}}$ and from $T_{c}^{s}$ to $T_{c}^{\mathrm{I}}$ and $T_{c}^{\mathrm{II}}$.

Now we examine in more detail how the color-flavor unlocking in case (c) proceeds with increasing $T$ from the region below $T_{c}^{\mathrm{I}}$.

(i) Just below $T_{c}^{\mathrm{I}}$, we have a CFL-like phase, but the three gaps take different values, with an order $\Delta_{3}>\Delta_{1}>$ $\Delta_{2} \neq 0$ (the mCFL phase). The reason why this order is realized can be understood from the GL potential (10). The $\epsilon$-term and $\eta$-term in Eq. (10) tend to destabilize us pairing $\left(\Delta_{2}\right)$ relative to $u d$ pairing $\left(\Delta_{3}\right)$ and $d s$ pairing $\left(\Delta_{1}\right)$. Since $\epsilon>\eta(>0), d s$ pairing is destabilized more effectively than $u d$ pairing. The value of each gap in the mCFL phase reads

$$
\begin{aligned}
& \Delta_{3}^{2}=\frac{\alpha_{0}}{8 \beta}\left(\frac{T_{c}-T}{T_{c}}+\frac{8}{3} \sigma\right), \\
& \Delta_{1}^{2}=\frac{\alpha_{0}}{8 \beta}\left(\frac{T_{c}-T}{T_{c}}-\frac{4}{3} \sigma\right), \\
& \Delta_{2}^{2}=\frac{\alpha_{0}}{8 \beta}\left(\frac{T_{c}-T}{T_{c}}-\frac{16}{3} \sigma\right) .
\end{aligned}
$$

The mCFL phase has only a global symmetry $U(1)_{C+L+R} \times U(1)_{C+L+R}$ in contrast to the global symmetry $S U(3)_{C+L+R}$ in the CFL phase with $m_{u, d, s}=0$. There are no gapless quark excitations in both mCFL and CFL phases. As $T$ increases, the first unlocking transition, the unlocking of $\Delta_{2}$ (the pairing between $B u$ and $R s$ quarks), takes place at the critical temperature,

$$
T_{c}^{\mathrm{I}}=\left(1-\frac{16}{3} \sigma\right) T_{c}
$$


(ii) For $T_{c}^{\mathrm{I}}<T<T_{c}^{\mathrm{II}}, \Delta_{2}=0$ and

$$
\begin{aligned}
& \Delta_{3}^{2}=\frac{\alpha_{0}}{6 \beta}\left(\frac{T_{c}-T}{T_{c}}+\frac{2}{3} \sigma\right), \\
& \Delta_{1}^{2}=\frac{\alpha_{0}}{6 \beta}\left(\frac{T_{c}-T}{T_{c}}-\frac{7}{3} \sigma\right) .
\end{aligned}
$$

In this phase, we have only $u d$ and $d s$ pairings (the dSC phase), and there is a manifest symmetry, $U(1)_{C+L+R} \times$ $U(1)_{C+L+R} \times U(1)_{C+V+B} \times U(1)_{C+V+B}$. By diagonalizing the inverse quark propagator in the Nambu-Gor'kov representation, we find three gapless quark excitations in the color-flavor combinations: $B u, R s$, and a linear combination of $R u$ and $B s$. At $T=T_{c}^{\mathrm{II}}$, the second unlocking transition, the unlocking of $\Delta_{1}$ (the pairing between $G s$ and $B d$ quarks), takes place at the critical temperature,

$$
T_{c}^{\mathrm{II}}=\left(1-\frac{7}{3} \sigma\right) T_{c}
$$

(iii) For $T_{c}^{\mathrm{II}}<T<T_{c}^{\mathrm{III}}$, one finds the $2 \mathrm{SC}$ phase, which has only $u d$ pairing with

$$
\Delta_{3}^{2}=\frac{\alpha_{0}}{4 \beta}\left(\frac{T_{c}-T}{T_{c}}-\frac{1}{3} \sigma\right) .
$$

The 2 SC phase has a symmetry $S U(2)_{C} \times S U(2)_{L+R} \times$ $U(1)_{C+B} \times U(1)_{L+R+B}[6]$. In this phase the $s$ quark and $B$ quark excitations are gapless. The final unlocking transition where $\Delta_{3}$ (the pairing between $R d$ and $G u$ quarks) vanishes occurs at

$$
T_{c}^{\mathrm{III}}=\left(1-\frac{1}{3} \sigma\right) T_{c}
$$

Above $T_{c}^{\mathrm{III}}$, the system is in the normal phase.

In Table I, we summarize the symmetry and the gapless quark modes in each phase discussed above. The number of gluons having nonzero Meissner masses, which is related to the remaining color symmetry, is also shown 14]. We note that more gapless quark modes may appear if the system is in the close vicinity of $T_{c}^{\mathrm{I}}, T_{c}^{\mathrm{II}}$, and $T_{c}^{\mathrm{III}}$ where $\Delta_{2}, \Delta_{1}$, and $\Delta_{3}$ are less than $\sim m_{s}^{2} / \mu[13$.

TABLE I: The symmetry, the gapless quark modes, and the number of Meissner screened gluons in the mCFL, dSC, and 2SC phases. The gapless quark mode $(R u, B s)$ denotes the linear combination of $R u$ and $B s$ quarks.

\begin{tabular}{|c|c|c|c|}
\hline & Symmetry & $\begin{array}{c}\text { Gapless } \\
\text { quark modes }\end{array}$ & $\begin{array}{c}\text { Number of } \\
\text { massive gluons }\end{array}$ \\
\hline \hline mCFL & {$[U(1)]^{2}$} & none & 8 \\
\hline $\mathrm{dSC}$ & {$[U(1)]^{4}$} & $\begin{array}{c}B u, R s \\
(R u, B s)\end{array}$ & 8 \\
\hline 2SC & {$[S U(2)]^{2} \times[U(1)]^{2}$} & $\begin{array}{c}B u, B d, B s, \\
R s, G s\end{array}$ & 5 \\
\hline
\end{tabular}

So far, we have studied the phase transitions in the mean-field level. In weak coupling, as shown in Ref. [6] in the massless limit, thermally fluctuating gauge fields could change the order of the transitions described in Figs. 1 and 2. A detailed account on this effect will be reported elsewhere [14]; here we recapitulate the important results. First, the second order transition, mCFL $\rightarrow \mathrm{dSC}$, remains second order even in the presence of the thermal gluon fluctuations. This is because all eight gluons are Meissner screened at $T=T_{c}^{\mathrm{I}}$ and thus cannot induce a cubic term with respect to the order parameter in the GL potential. On the other hand, the transitions, $\mathrm{dSC} \rightarrow 2 \mathrm{SC}$ and $2 \mathrm{SC} \rightarrow$ normal, become weak first order since some gluons, which are massless in the high temperature phase, become Meissner screened in the low temperature phase (Table I).

In summary, we have investigated color-flavor unlockings at finite temperatures taking into account the strange quark mass and charge neutrality in the GL approach. We find three successive unlocking transitions, $\mathrm{mCFL} \rightarrow \mathrm{dSC} \rightarrow 2 \mathrm{SC} \rightarrow$ normal, occurring in weak coupling. Most remarkably, the dSC phase appears between the mCFL and 2SC phases. In this phase all eight gluons are Meissner screened and the three quark excitations are gapless. The question of how the phase structure of neutral quark matter obtained near $T_{c}$ is connected to that at $T=0$, which approaches the CFL phase in the limit of high density, is an interesting open problem.

We are grateful to G. Baym and H. Abuki for helpful discussions. This work was supported in part by RIKEN Special Postdoctoral Researchers Grant No. A11-52040 and No. A12-52010, and by the Grants-in-Aid of the Japanese Ministry of Education, Culture, Sports, Science, and Technology (No. 15540254).

[1] D. Bailin and A. Love, Phys. Rep. 107, 325 (1984); M. Iwasaki and T. Iwado, Phys. Lett. B 350, 163 (1995); M. Alford, K. Rajagopal, and F. Wilczek, Phys. Lett. B 422, 247 (1998); R. Rapp, T. Schäfer, E.V. Shuryak, and M. Velkovsky, Phys. Rev. Lett. 81, 53 (1998).

[2] For reviews, see K. Rajagopal and F. Wilczek, in Handbook of $Q C D$, edited by M. Shifman (World Scientific, Singapore, 2001); M.G. Alford, Ann. Rev. Nucl. Part. Sci. 51, 131 (2001).

[3] S. Reddy, M. Sadzikowski, and M. Tachibana, Nucl. Phys. A714, 337 (2003), and references therein.

[4] K. Iida and G. Baym, Phys. Rev. D 63, 074018 (2001); ibid. 66, 059903(E) (2002).

[5] K. Iida and G. Baym, Phys. Rev. D 66, 014015 (2002).

[6] T. Matsuura, K. Iida, T. Hatsuda, and G. Baym, Phys. Rev. D (in press); hep-ph/0312042.

[7] A.W. Steiner, S. Reddy, and M. Prakash, Phys. Rev. D 66, 094007 (2002).

[8] H. Abuki, Prog. Theor. Phys. 110, 937 (2003).

[9] D.T. Son, Phys. Rev. D 59, 094019 (1999); D.K. Hong, Nucl. Phys. B582, 451 (2000); R.D. Pisarski and D.H. 
Rischke, Phys. Rev. D 61, 074017 (2000); W.E. Brown, J.T. Liu, and H.C. Ren, Phys. Rev. D 62, 054016 (2000). [10] R.D. Pisarski, Phys. Rev. C 62, 035202 (2000).

[11] D.D. Dietrich and D.H. Rischke, nucl-th/0312044.

[12] T. Schäfer, Phys. Rev. D 65, 094033 (2002).

[13] I. Shovkovy and M. Huang, Phys. Lett. B 564, 205
(2003); M. Huang, and I. Shovkovy, Nucl. Phys. A729, 835 (2003); M. Alford, C. Kouvaris, and K. Rajagopal, hep-ph/0311286.

[14] T. Matsuura, K. Iida, M. Tachibana, and T. Hatsuda (unpublished). 